\title{
Deployable Tensegrity Reflectors for Small Satellites
}

\author{
A. G. Tibert* and S. Pellegrino ${ }^{\dagger}$ \\ University of Cambridge, Cambridge, England CB2 1PZ, United Kingdom
}

\begin{abstract}
Future small satellite missions require low-cost, precision reflector structures with large aperture that can be packaged in a small envelope. Existing furlable reflectors form a compact package which, although narrow, is too tall for many applications. An alternative approach is proposed, consisting of a deployable "tensegrity" prism forming a ring structure that deploys two identical cable nets (front and rear nets) interconnected by tension ties; the reflecting mesh is attached to the front net. The geometric configuration of the structure has been optimized to reduce the compression in the struts of the tensegrity prism. A small-scale physical model has been constructed to demonstrate the proposed concept. A preliminary design of a 3-m-diam, 10-GHz reflector with a focal-length-todiameter ratio of 0.4 that can be packaged within an envelope of $0.1 \times 0.2 \times 0.8 \mathrm{~m}^{3}$ is presented.
\end{abstract}

\section{Nomenclature}

$\begin{array}{ll}A & =\text { area, } \mathrm{m}^{2} \\ a & =\text { radius of tube cross section, } \mathrm{m} \\ b & =\text { number of bars } \\ D & =\text { aperture diameter, } \mathrm{m} \\ E & =\text { Young's modulus, } \mathrm{GN} / \mathrm{m}^{2} \\ F & =\text { focal length, } \mathrm{m} \\ H & =\text { depth, } \mathrm{m} \\ j & =\text { number of joints } \\ L & =\text { triangle side length, } \mathrm{m} \\ L_{e} & =\text { effective length, } \mathrm{m} \\ m & =\text { number of independent mechanisms } \\ N & =\text { mesh tension per unit length, } \mathrm{N} / \mathrm{m} \\ R & =\text { radius of approximating sphere, } \mathrm{m} \\ r & =\text { radius of gyration, } \mathrm{m} \\ S & =\text { number of independent states of self-stress } \\ T & =\text { tension, } \mathrm{N} \\ \delta_{\text {rms }} & =\text { root-mean-square surface error, } \mathrm{mm} \\ \theta & =\text { relative rotation between nets, deg } \\ \rho & =\text { density, kg/m }{ }^{3}\end{array}$

\section{Introduction}

$\mathbf{T}$ HERE is currently a growing interest in low-cost deployable appendages for small satellites. The work presented in this paper was motivated by an application requiring a 3-m-diam parabolic reflector to be launched alongside a $0.6 \times 0.6 \times 0.8 \mathrm{~m}^{3}$ bus, packaged within an envelope of $0.1 \times 0.2 \times 0.8 \mathrm{~m}^{3}$. This reflector has a focallength to diameterratio $F / D=0.4$ and operates at a frequency of $10 \mathrm{GHz}$. When inflatables are excluded, which still cannot be regarded as a mature technology, none of the existing deployable reflector concepts can meet these requirements. It was considered that the greatest potential for meeting the requirements with a lowcost system would be offered by an adaptation of the AstroMesh concept. ${ }^{1,2}$

In the AstroMesh (Fig. 1), the reflective mesh is attached to a network of thin cables, or tapes, with high axial stiffness that approximates to a paraboloid; the cables are prestressed to form a stiff and accurate structure. The size of the triangles forming the cable

Presented as Paper 2001-1261 at the AIAA Gossamer Spacecraft Forum, Seattle, WA, 16-19 April 2001; received 19 November 2001; revision received 29 March 2002; accepted for publication 18 April 2002. Copyright (C) 2002 by A. G. Tibert and S. Pellegrino. Published by the American Institute of Aeronautics and Astronautics, Inc., with permission. Copies of this paper may be made for personal or internal use, on condition that the copier pay the $\$ 10.00$ per-copy fee to the Copyright Clearance Center, Inc., 222 Rosewood Drive, Danvers, MA 01923; include the code 0022-4650/02 \$10.00 in correspondence with the CCC.

*Junior Visitor, Department of Engineering; currently Researcher, Department of Mechanics, Royal Institute of Technology, SE 10044 Stockholm, Sweden.

${ }^{\dagger}$ Professor of Structural Engineering, Department of Engineering, Trumpington Street. Associate Fellow AIAA. network is chosen sufficiently small to achieve the required accuracy. This concept is known as a tension truss and was invented by Miura. ${ }^{3}$ The forces required to prestress the cable net are provided by a series of springs, called tension ties in Fig. 1, connecting the network to an identical rear net. Both nets are connected around the edge to a deployable ring truss with telescopic diagonals.

Note that the height of the ring truss is given by the depth of the two nets plus their separation. Although the rear net can be made less deep, for example, half the depth of the front net, by accepting larger forces on the ring, a reflector with small $F / D$ requires a higher ring. For example, for depth $H=0.46 \mathrm{~m}$ and $D=3 \mathrm{~m}$, an AstroMeshtype truss divided into 18 segments would have a packaged height of more than $1.2 \mathrm{~m}$. An alternative ring configuration based on a pantograph with, again, 18 bays would have a height of $0.9 \mathrm{~m}$. However, this requires a much larger number of joints.

A new concept that meets these requirements is presented in the next section; then a detailed analysis of the prestress distribution in the reflector structure is carried out, considering the effects of several design parameters. This leads to a preferred configuration of the reflector, whose feasibility has been demonstrated by constructing a physical model. A preliminary design of a 3-m reflector that would meet all of the requirements of the particular application that motivated the present study is then presented.

\section{New Concept}

The proposed reflector structure is based on the tension truss concept. Like the AstroMesh, it is composed of three main parts: a deployable ring structure, two identical cable nets (front and rear nets) connected by tension ties, and the reflecting mesh, attached to the front net.

Although the concept is a general one, for clarity it will be explained with reference to the particular example shown in Fig. 2. Here, every member is a cable element, apart from six struts, drawn with thick lines in Fig. 2a and a series of constant-tension springs, called tension ties in Fig. 2 d.

Figure 2a highlights the 18 cable elements and 6 struts that form the deployable ring structure. This well-known "tensegrity structure" belongs to a family invented in 1948 by Snelson and Buckminster Fuller. The potential usefulness of deployable tensegrity structures for spacecraft applications has been pointed out several times, most recently in Ref. 4. Two important features of tensegrity structures are that there is no connection between compression elements and that the connections between compression and tension members are simple to manufacture. Also, their joints are not affected by friction or dead bands and, hence, behave linearly.

These features make them particularly attractive for applications requiring low-weight, low-cost deployables that can be packaged very compactly. A disadvantage of standard tensegrity structures is that they are very flexible, due to the existence of internal mechanisms of inextensionaldeformation, as will be shown next. However, the solution then presented avoids this problem. 


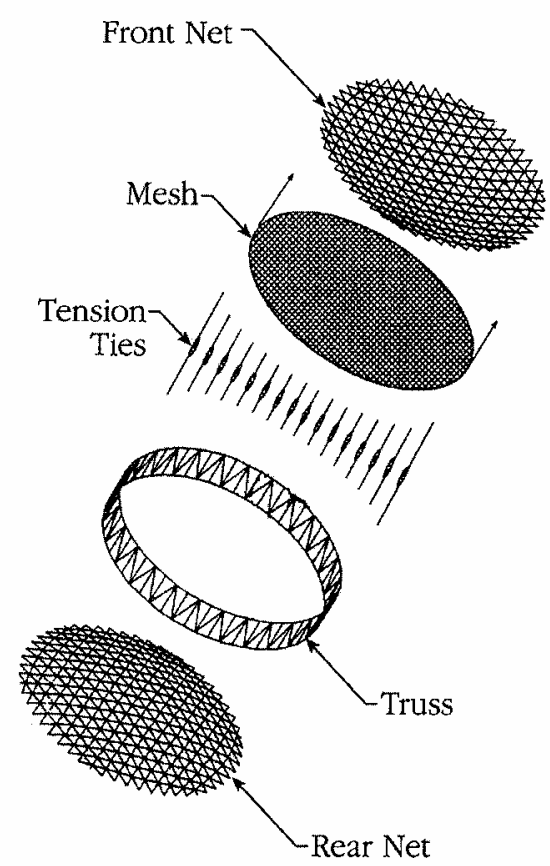

Fig. 1 AstroMesh concept (from Thomson ${ }^{1}$ ).

Consider the pin-jointed structure shown in Fig. 3, whose layout is identical to the ring structure in Fig. 2. The top six joints lie at the corners of a regular hexagon, and the bottom six joints lie at the corners of an identical hexagon. Consider a joint in the bottom hexagon; it is connected by bars to the two neighboring joints in the same hexagon as well as to two joints of the top hexagon. Note that it is not connected to the joint directly above, but to the next and the second next joints, in an anticlockwise sense.

This structure has $j=12$ joints and $b=24$ bars. The static and kinematic properties of a general pin-jointed structure can be analysed using the extended Maxwell's rule (see Ref. 5),

$$
3 j-b=m-s
$$

where $m$ is the number of independent inextensional mechanisms and $s$ is the number of independent states of self-stress.

Substituting the values of $j$ and $b$ into Eq. (1), we obtain

$$
m-s=12
$$

It can be shown, for example, by computing all nontrivial solutions of the system of equations of joint equilibrium, that this structure has one state of self-stress, $s=1$, where the six longer bars connecting the two hexagons are in compression and all other members are in tension. Therefore, from Eq. (2), we conclude that $m=13$ and, because 6 mechanisms will involve rigid-body motions of the whole structure, this leaves 7 internal mechanisms. These mechanisms can be stiffened by prestressing the structure, but this will provide only a relatively small stiffness.

Because this structure can be prestressed, as explained earlier, a deployable version could be made quite easily: The state of prestress requires only six members to carry compressive forces, all other members are in tension, and therefore, instead of using bars, cables can be used. Then, if the struts are collapsible, the whole structure can be folded.

This structure, however, has seven internal mechanisms, which would result in seven independent "soft" modes of deformation. An alternative configuration that is practically more useful is shown in Fig. 4. This structure is obtained by connecting the nodes of the top and bottom hexagons to two interconnected,central joints. Note that these internal joints are not coplanar with the hexagons, thus forming two triangulated surfaces that coarsely approximate to a curved surface. This structure is a simplified version of the new concept shown in Fig. 2.

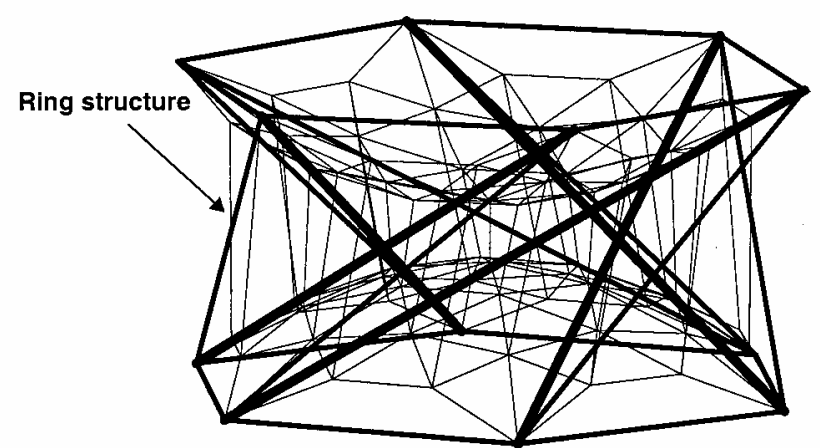

a)

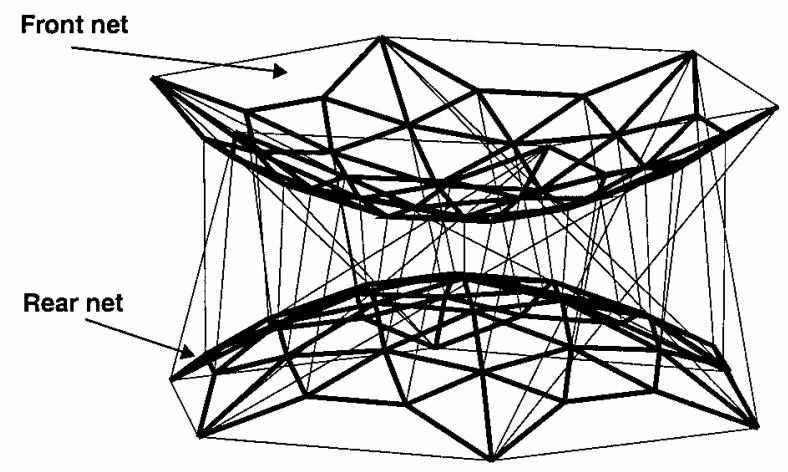

b)

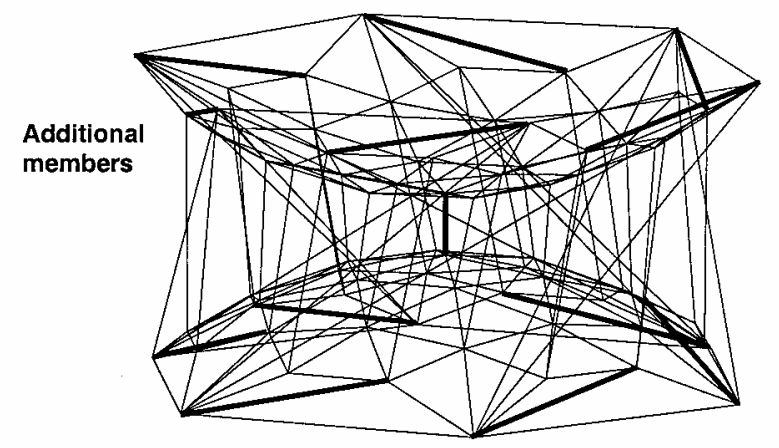

c)

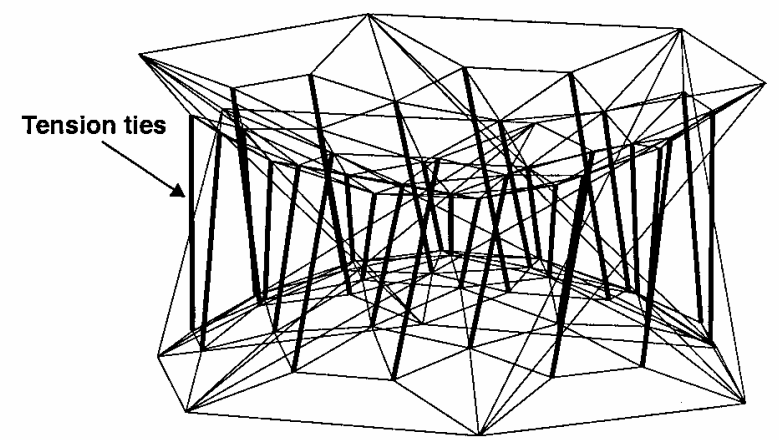

d)

Fig. 2 New furlable reflector concept.

The number of joints and bars are, respectively, $j=14$ and $b=37$. Hence, Eq. (1) gives

$$
m-s=5
$$

and, because the same state of self-stress of the structure in Fig. 3 exists also for this structure and there is no other independent state of self-stress, $s=1$ as before. Hence, $m=6$, and so this structure has only six rigid-body mechanisms. Because it is internally rigid, this structure is potentially useful for high-precision applications. However, the triangulated surfaces in this example are far too coarse to support a reflective mesh that approximates to a paraboloid. 


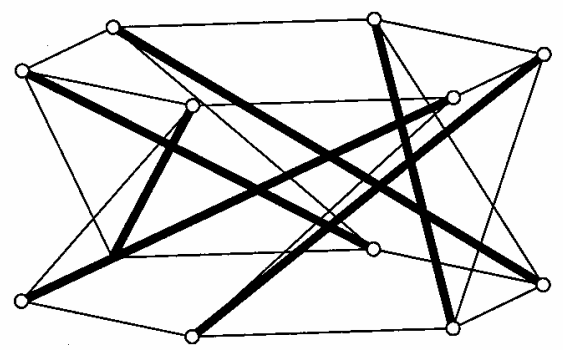

a) Three-dimensional view

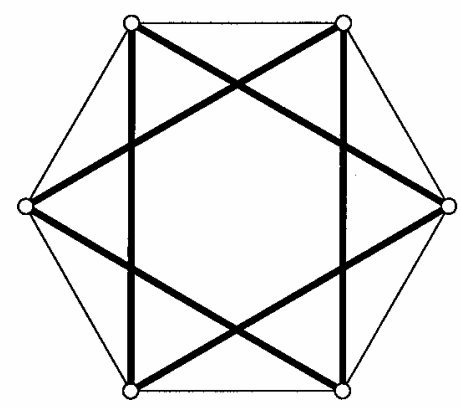

b) Top view

Fig. 3 Hexagonal tensegrity module.

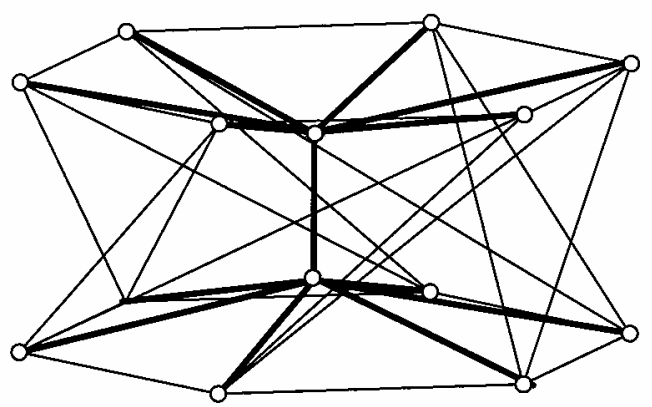

Fig. 4 Hexagonal tensegrity module with interconnected front and rear triangulated structures.

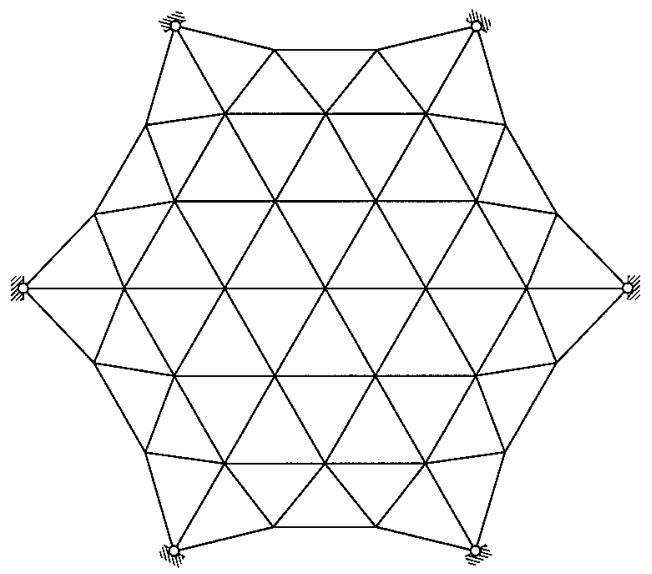

Fig. 5 Layout of front and rear nets.

Hence, these simple triangulated surfaces will be replaced with the net shown in Fig. 5.

The layout of a triangulated net can be defined in many different ways; for example, it could be optimized such that all triangles have equal areas and are as close as possible to equilateral. The particular layout that was chosen is based on a simple two-dimensional,regular tessellation of equilateral triangles that is obtained by dividing each side of a hexagon into three. Then, the outermost triangles were distorted to form a catenarylike edge for the net, to improve the force distribution. Finally, all of the nodes were projected onto a paraboloid. Further details are given in the Appendix.
Now, consider the structure consisting of the original ring structure plus the two triangulatednets; its static and kinematic properties are investigatedas follows. First, there are six joints in the symmetry unit of each net; hence, in total

$$
j=2 \times(1+6 \times 6)=74
$$

Second, there are 15 bars in the symmetry unit of each net, plus the 24 bars of the ring structure; hence, in total

$$
b=2 \times(15 \times 6)+24=204
$$

Substituting Eqs. (4) and (5) into Eq. (1), we obtain

$$
m-s=18
$$

Because the state of self-stress describedearlier is still staticallypossible, but no additional states of self-stress have been created, $s=1$. Hence, $m=19$, of which 6 mechanisms are rigid-body motions and 13 are internal mechanisms. The 13 internal mechanisms can be removed by adding 13 bars to the structure, as shown in Fig. 2c. The resulting structure has $m=6$, and, hence, all mechanisms are rigid-body mechanisms, and $s=1$.

To transform this pin-jointed structural concept into an efficient deployable structure made from cable elements, we need to find a way of prestressing the two nets once they have taken up their fully deployed shape. An obvious approach, based on the AstroMesh, might be to connect correspondingnodes of the two nets with tension ties, but it turns out that this is not an ideal solution because large compressive forces are induced in the cables of the ring structure, which need to be counteracted by increasing the level of prestress of the ring. This would further increase the compression in the struts; also, 12 of the 13 additional members shown in Fig. 2c are not pretensioned.

Both of these issues can be resolved by modifying the configuration of the ring structure. Instead of using the original configuration, where the two hexagons are directly one above the other, as highlighted by Fig. 3b, one hexagon is rotated through a small angle $\theta$, as shown in Fig. 6 . Note that $\theta$ is defined to be positive if the upper hexagon is rotated anticlockwise with respect to the bottom hexagon.

By itself, the resulting ring structure can no longer be prestressed, as $s=0$, and hence, from Eq. (2), $m=12$. However, when the complete reflector structure is considered, including the prestressing forces applied by the tension ties, it is found that there are only 12 internal mechanisms, and hence, the additional member connecting the centers of the two nets can be replaced with a tension tie. Also, for $\theta=+10 \mathrm{deg}$, as shown in Fig. 6 , all of the cables are in a state of tension. Note that the forces in the outer ties have been set to twice the value of the internal ties.

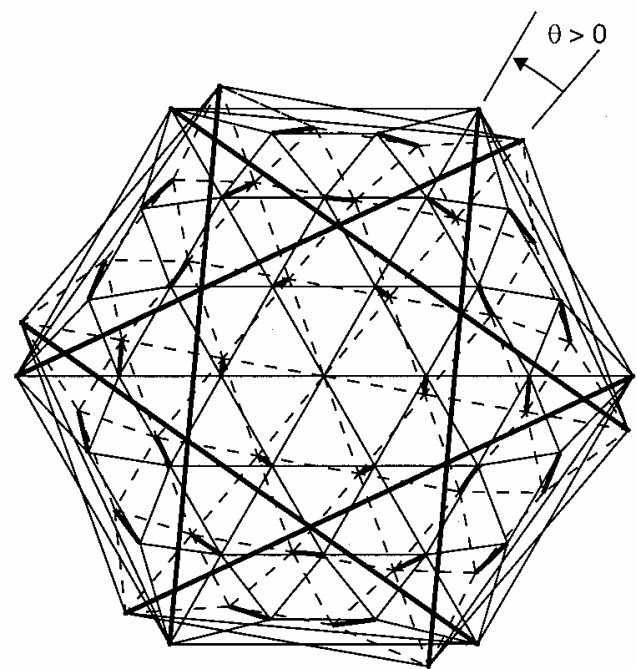

Fig. 6 Complete structure, but without additional 12 members: front net; - - - , rear net; $\longrightarrow$, tension ties; - , struts; and $\longrightarrow$, ring cords. 


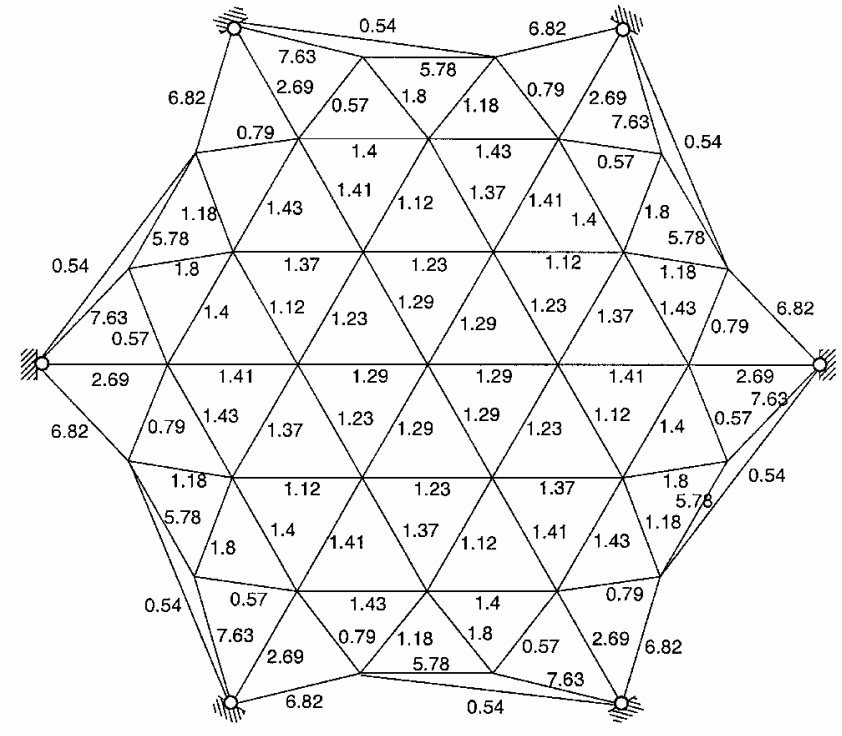

Fig. 7 Forces in the two nets due to tension tie loads of $1 \mathrm{~N}$ on the inner nodes and $2 \mathrm{~N}$ on the edge nodes.

Figure 7 shows the force distribution in the two nets: The corresponding forces in the ring structure are $-68.8 \mathrm{~N}$ in the struts, $+25.9 \mathrm{~N}$ in the cables forming the hexagons, and $+39.5 \mathrm{~N}$ in the six cables linking the hexagons. Hence, the ratio between the smallest and largest force in the structure is $0.54 / 68.8=0.78 \%$.

\section{Configuration of Tensegrity Reflector}

In addition to studying the statical and kinematical properties of the reflector structure, it is necessary to analyze the effect of different design parameters on the magnitude and distribution of the forces within the structure. The aim of this study is to obtain a fairly uniform distribution of forces in the front net and to avoid large forces in the supporting structure, particularly the struts. Hence, the configuration study presented in this section is divided into two parts. First, the influences of the sag of the edges of the net and of the tension tie forces on the force distribution are investigated, without considering the rest of the structure. Then, for some particular values of the forces in the tension ties and a particular sag-to-span ratio, the effect of the relative rotation of the hexagons on the forces in the nets and the ring structure is investigated. A detailed description of the procedure used for generating the triangular net and a definition of the sag-to-span ratio are given in the Appendix.

\section{Sag-to-Span Ratio and Tension Tie Forces}

Consider the cable net shown in Fig. 8, where the tension ties are represented by vertical loads applied to the joints.

First, its statical and kinematical properties will be checked. The number of joints is (see the Appendix)

$$
j=1+6 \times 3(1+3) / 2=37
$$

and the number of bars is

$$
b=6 \times 3(1+3 \times 3) / 2=90
$$

hence, Eq. (1) yields

$$
m-s=21
$$

Because the net has synclastic shape, that is, principal curvatures of equal signs, it is obvious that no state of self-stress is possible; hence, $s=0$. As shown in Fig. 8, 21 constraints are added, 18 by fixing completely 6 joints around the edge of the net. The remaining three constraints fix one edge joint both radially and tangentially, that is, in two perpendicular directions, and an adjacent edge joint radially. The arrangement of these constraints is such that the three mechanisms of the structure shown in Fig. 8 are removed; these

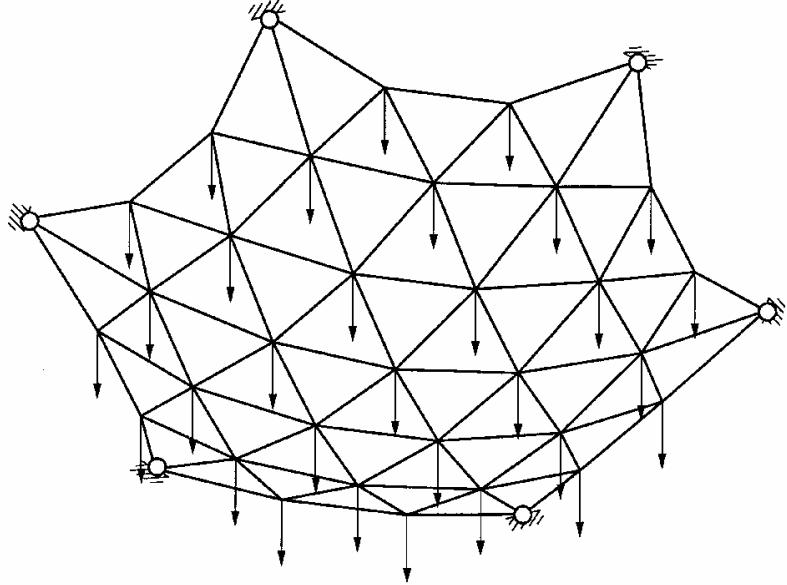

Fig. 8 Loads applied to cable net.

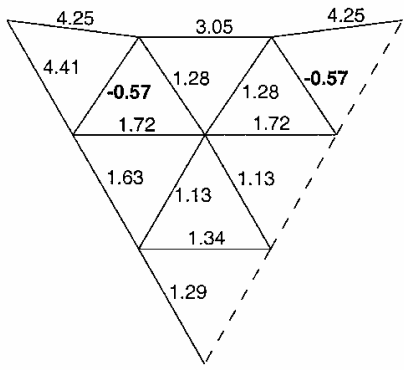

a) $1 \mathrm{~N}$

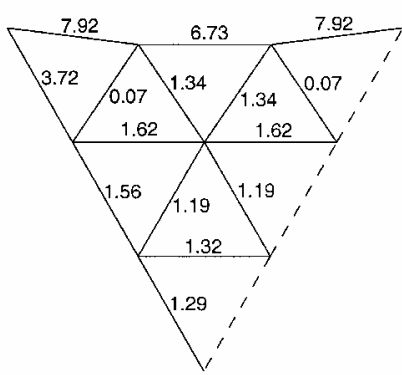

b) $2 \mathrm{~N}$

Fig. 9 Forces in a net with $5 \%$ sag-to-span ratio; loads on inner nodes $1 \mathrm{~N}$ and loads on edge nodes as shown.

mechanisms were computed from the equilibrium matrix of the structure. ${ }^{6}$

This produced a statically and kinematically determinate structure, that is, $s=0$ and $m=0$, that was analyzed for three sag-to-span ratios: 5,10 and $15 \%$. For each ratio, the tension tie forces were initially set to $1 \mathrm{~N}$ everywhere; this would be easy to realize in practice, with identical constant-tension springs in all of the tension ties.

The results for a 5\% sag-to-span ratio are shown in Fig. 9. When the tension tie forces are all equal to $1 \mathrm{~N}$ (Fig. 9a), some members are in compression. When the edge tie forces are increased, the compressive forces gradually become smaller and then tensile (Figs. 9b-9d). An almost uniform force distribution is obtained for edge forces of $4 \mathrm{~N}$; however, the largest force in the edge cable is now over $15 \mathrm{~N}$.

When the sag-to-span ratio is increased to $10 \%$, there is still compression for tension tie forces of $1 \mathrm{~N}$ (Fig. 10a). However, as the edge tie forces are increased to $2 \mathrm{~N}$, an acceptable distribution of forces is obtained, and the edge cable forces are smaller than for the $5 \%$ sag-to-span ratio (Fig. 10b). The range of the inner net forces is $0.75-2.69 \mathrm{~N}$.

Increasing the sag-to-span ratio further, to $15 \%$, yields no compressed elements even for the case of uniform 1-N tension tie loads (Fig. 11a). When increased to $2 \mathrm{~N}$, the forces in the edge ties give a very uniform force pattern, in the range $1.27-2.08 \mathrm{~N}$, and the edge cable forces are slightly smaller than in the preceding case. Although 

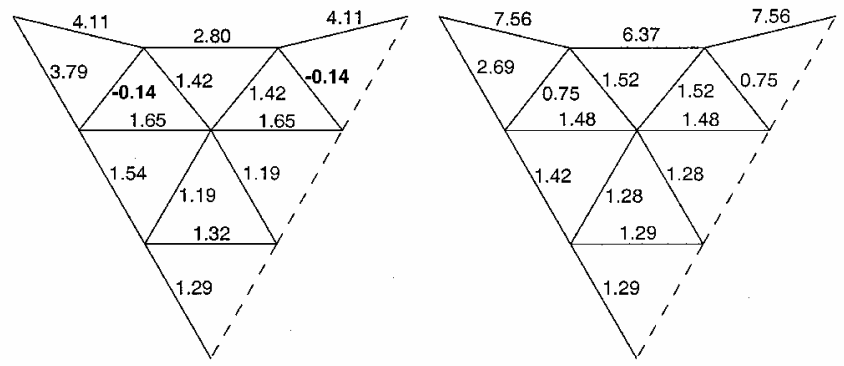

a) $1 \mathrm{~N}$

b) $2 \mathrm{~N}$

Fig. 10 Forces in a net with $10 \%$ sag-to-span ratio; loads on inner nodes $1 \mathrm{~N}$ and loads on edge nodes as shown.
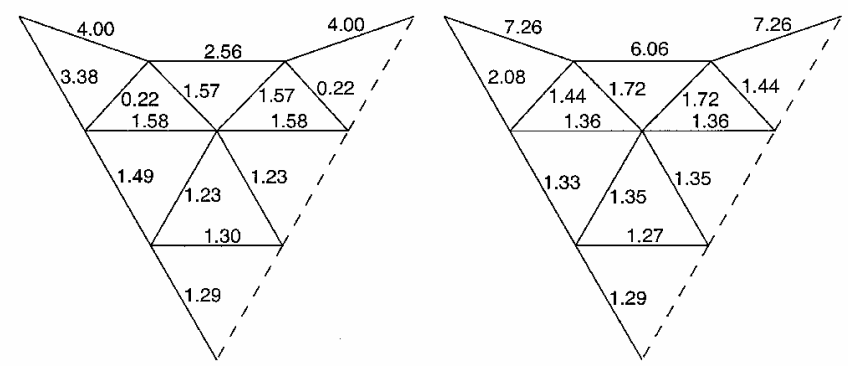

a) $1 \mathrm{~N}$

b) $2 \mathrm{~N}$

Fig. 11 Forces in a net with $15 \%$ sag-to-span ratio; loads on inner nodes $1 \mathrm{~N}$ and loads on edge nodes as shown.

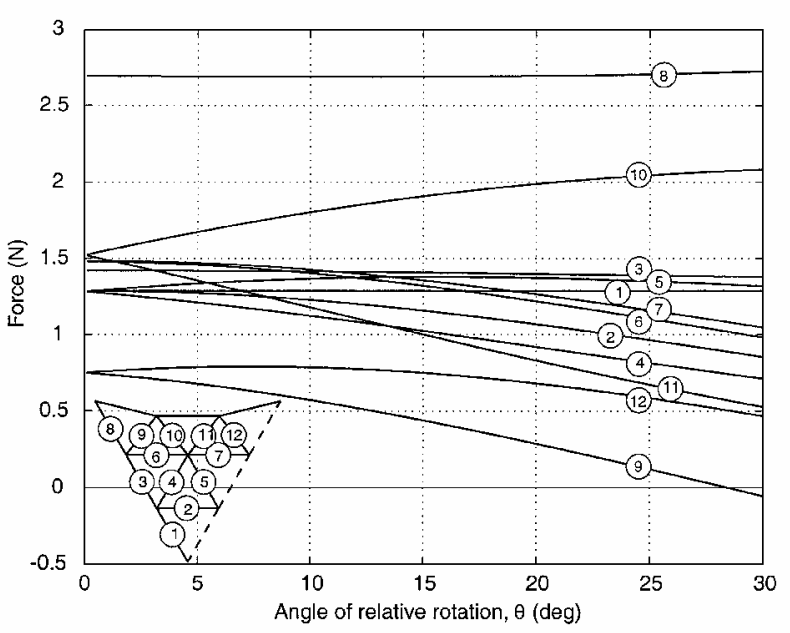

Fig. 12 Variation of forces in net cables.

a sag-to-span ratio of $15 \%$ gives a better force pattern than the $10 \%$ ratio, the further reduction in the surface area of the net, and hence of the reflecting surface of the reflector, is not justified; thus, a value of $10 \%$ was selected.

\section{Rotation of Hexagons}

The effect of a relative rotation $\theta$ between the hexagons of the ring structure was analyzed, for cable nets with a sag-to-spanratio of $10 \%$. The tension tie forces were taken to be $1 \mathrm{~N}$ on the inner joints and $2 \mathrm{~N}$ on the edge joints. The corresponding force distribution for $\theta=0 \mathrm{deg}$ is shown in Fig. 10b. When the hexagons are rotated, the force distribution in a symmetry unit of the net is no longer symmetric; see Figs. 12 and 13.

\section{Net Forces}

Figure 12 is a plot of the variation in the forces of the inner cables with the rotation of the hexagons. The forces in the radial cables 1, 3, and 8 are approximately constant for the range of $\theta$ displayed. The other cable forces, except for cables 9 and 10, are in the range $0.5-$ $1.5 \mathrm{~N}$. Most important, cable 9 becomes compressed at $\theta \approx 28 \mathrm{deg}$,

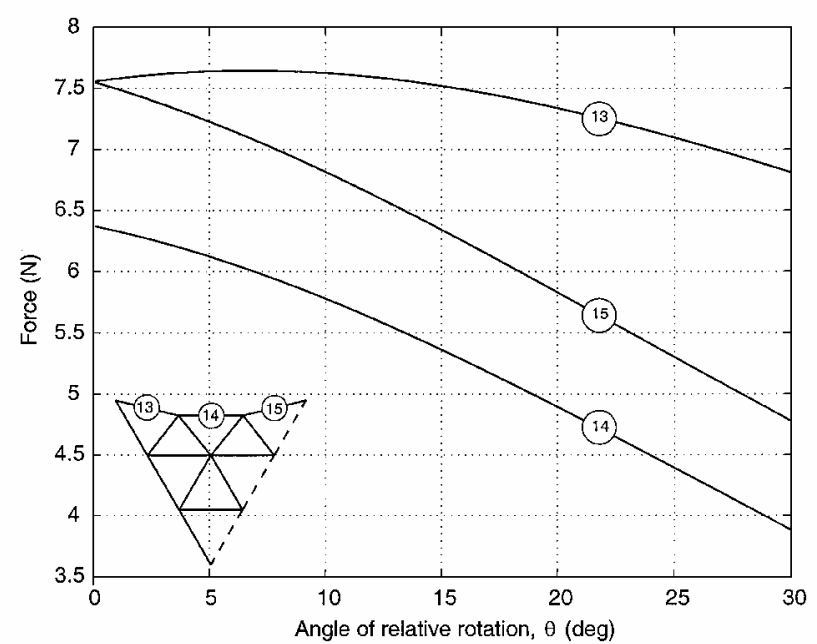

Fig. 13 Variation of forces in edge cables.

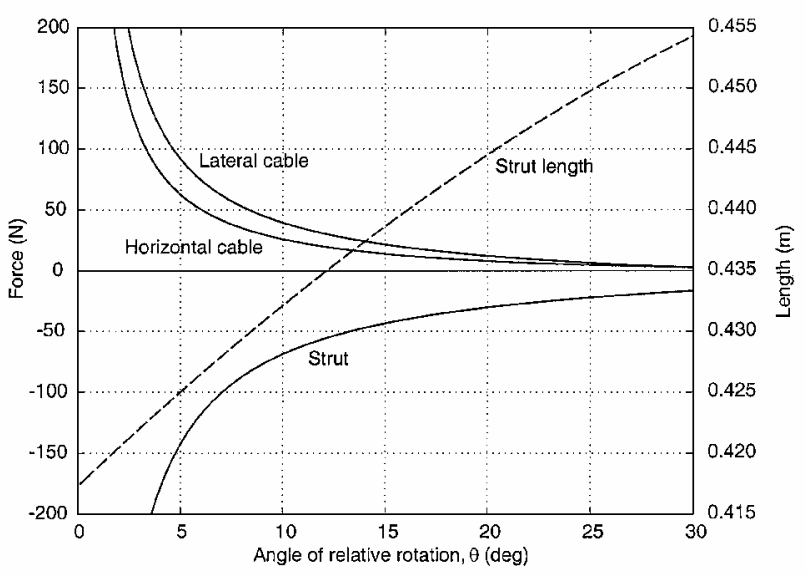

Fig. 14 Variation of forces in ring structure.

giving an upper limit on $\theta$ for the particular reflector configuration studied here.

Figure 13 is a plot of the variation in the forces of the edge elements. The force in edge cable 13 initially increases and then decreases. Edge forces 14 and 15 decrease when $\theta$ is increased. This is due to the change in the direction of the tension tie forces.

\section{Ring Forces}

The forcesin the ring structure vary exponentiallywith $\theta$ (Fig. 14). For small $\theta$, the forces are very large, especially in the struts, but decrease to acceptable levels for $\theta \approx 10 \mathrm{deg}$. Further rotation leads to a slow decrease in force magnitudes, and for the practical limit $\theta=28$ deg discussed earlier, the force in the lateral cables is $3.9 \mathrm{~N}$.

Because the structure is kinematically determinate, its stiffness depends on the elastic properties of its members and is independent of the prestress level. However, the cable pretensions must be sufficiently high that all cables remain in tension under the action of any compressive loads induced by the loads.

Also shown in Fig. 14 is the variation of the strut length, which is much smaller than the variation of the forces in the struts. Of course, shorter struts are preferable, to avoid buckling.

\section{Additional Members}

The 12 additional members have the function of removing all internal mechanisms, as explained in the section "New Concept." To use cable elements instead of solid bars, it must be ensured that these elements are pretensioned. Here an advantage of the configuration shown in Fig. $2 \mathrm{c}$ is that the additional elements are in tension for any $\theta>0$ deg (Fig. 15), and the magnitude of the tension increases almost linearly up to about $10 \mathrm{deg}$. If the additional members were 


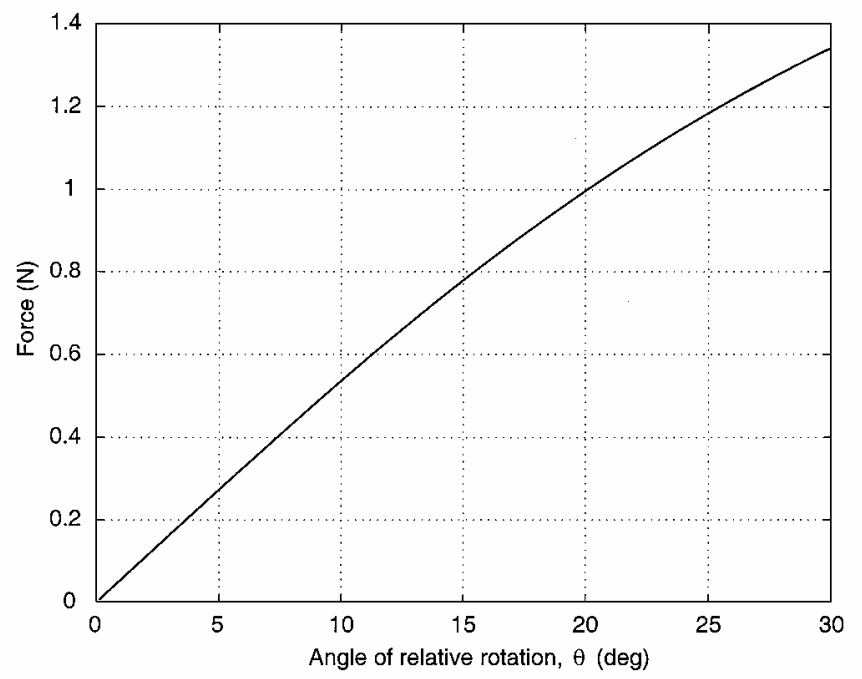

Fig. 15 Variation of forces in additional members.

rearranged, to turn in the opposite sense about the axis of the reflector, they would be in compression for $\theta>0 \mathrm{deg}$, instead.

\section{Other Issues}

Anotherimportantissue, not concerned with the force distribution within the structure, is that the struts move closer to the center of the reflector when $\theta$ is increased. Hence, the struts are more likely to interfere with the tension ties, which would complicate the deployment procedure. Therefore, it is important to $\operatorname{keep} \theta$ small.

\section{Demonstration Model}

To verify the feasibility of the proposed concept, a small-scale physical model was constructed, with a diameter of $0.47 \mathrm{~m}$. The triangulated nets were constructed on paraboloidal molds of PETG (a thermoplastic material with the trade name of Vivak) with diameter $D=0.45 \mathrm{~m}$ and focal length $F=0.134 \mathrm{~m}$, on which the position of the nodes of the net had been marked with a three-axis computercontrolled machine. The net was made from 0.8-mm-diam Kevlar ${ }^{\circledR}$ cords, which had been pretensioned before being taped to the mold; the cords were joined with nylon loops at all crossover points and bonded with epoxy resin. Corresponding nodes of the two nets were connected with steel springs.

Identical Al-alloy, 30-mm-long joint fittings, of cylindrical shape with a diameter of $15 \mathrm{~mm}$, were attached to the six corners of each net. Holes with $2.0 \mathrm{~mm}$ diam in the direction of each cord had been drilled in the joint fittings, and all connections were made with epoxy resin. The cords of the ring structure, consisting of 1.0-mm Kevlar cord, were connected to the same joint fittings.

The telescopic struts, $0.46 \mathrm{~m}$ long, were made by cutting off the sticks of six identical foldable umbrellas. The umbrella sticks were inserted into 20-mm-long, 6.4-mm-diam holes at the bottom of the joint fittings; these holes are coaxial with the joints. Grub screws hold the joints on the umbrella sticks.

This proof-of-conceptmodel works well, considering that it was the first time that a structure of this kind had been constructed in our laboratory. The model can be easily folded and deployed by hand, as shown in Figs. 16-18. However, some of the cables in the two nets remain slack after deployment, and there is some interference between the nets and the struts, because the diameter of the net, as manufactured, turned out to be bigger than expected. Correcting these problems should be possible in a second-generationmodel.

Figure 18 shows the very compact, packaged configuration of the reflector; note the elongated shape of the package, which would permit its stowage alongside the small satellite described in the Introduction.

\section{Preliminary Design of 3-m Reflector}

The main characteristics of a reflector to meet the requirements $D=3 \mathrm{~m}, F=1.2 \mathrm{~m}$, and operation at $10 \mathrm{GHz}$ are determined in

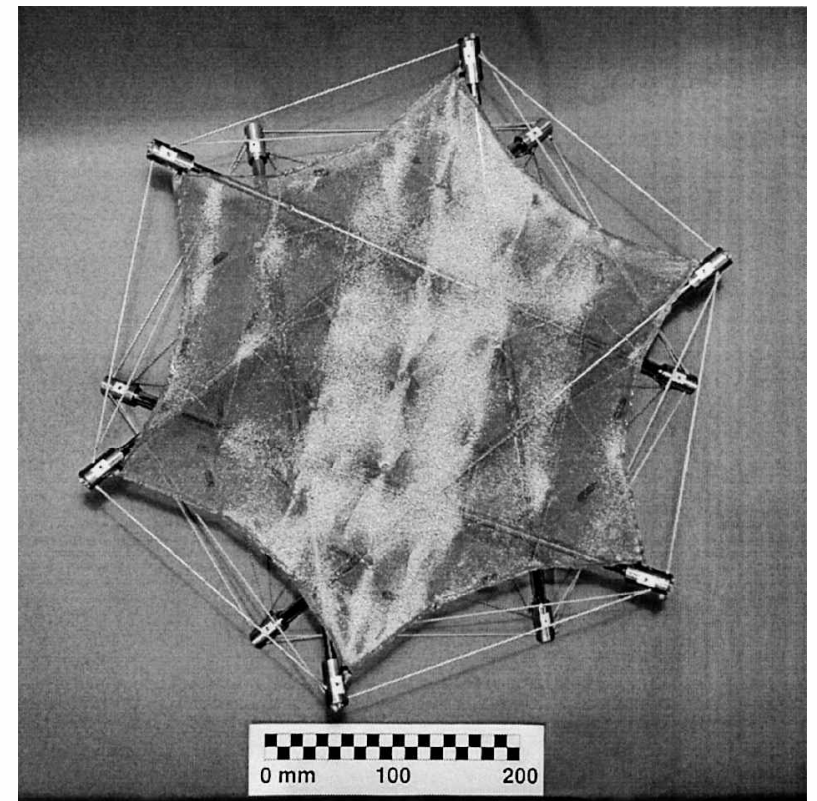

a)

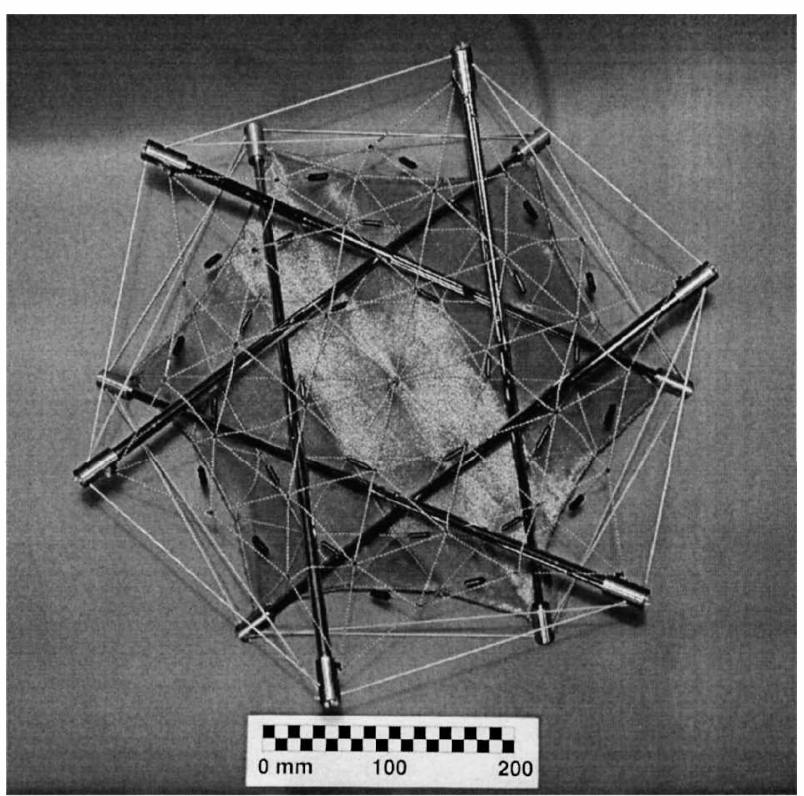

b)

Fig. 16 Model structure, expanded: view of a) top and b) bottom.

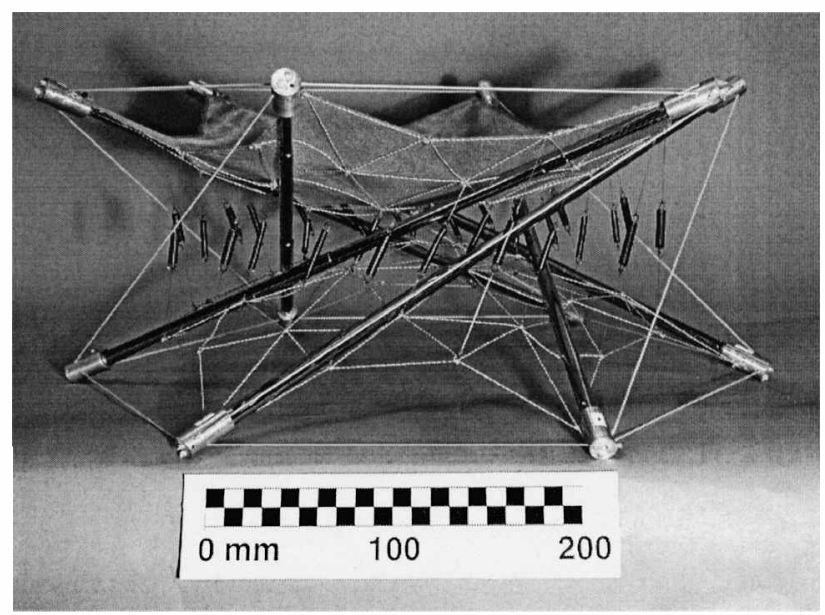

Fig. 17 Side view of model structure, expanded. 
Table 1 Mass estimates for 3-m-diam reflector

\begin{tabular}{lclc}
\hline \hline Element & Quantity & Unit mass & Mass, kg \\
\hline Net cables & $300 \mathrm{~m}$ & $0.0087 \mathrm{~kg} / \mathrm{m}$ & 1.04 \\
Struts & $17.3 \mathrm{~m}$ & $0.170 \mathrm{~kg} / \mathrm{m}$ & 2.94 \\
Ring cables & $29.8 \mathrm{~m}$ & $0.0066 \mathrm{~kg} / \mathrm{m}$ & 0.19 \\
Motors and latches & 6 & $0.20 \mathrm{~kg} / \mathrm{item}$ & 1.20 \\
Connections & 12 & $0.050 \mathrm{~kg} / \mathrm{item}$ & 0.60 \\
Mesh & $7.07 \mathrm{~m}^{2}$ & $0.050 \mathrm{~kg} / \mathrm{m}^{2}$ & 0.35 \\
Total & & & 6.32 \\
\hline \hline
\end{tabular}

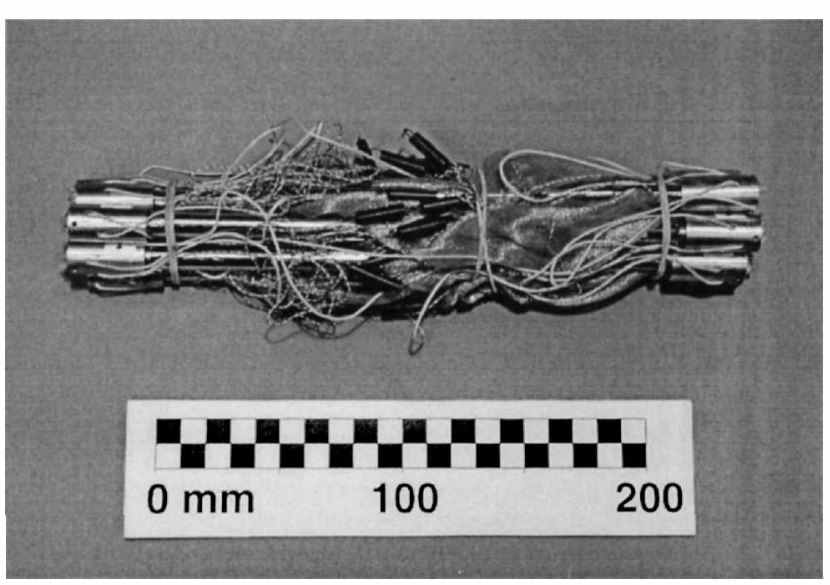

Fig. 18 Model structure, folded.

this section. In particular, an estimate of the mass of the reflector, summarized in Table 1, is obtained.

\section{Network Density}

The surface error of the reflector originates from a number of different sources, such as thermal distortion of the structure, etc. Only one contribution to the overall error budget can be considered at this stage, namely, the effect of approximating the required paraboloid with a polyhedral surface. Therefore, it will be conservatively required that the root-mean-squareerror $\delta_{\text {rms }}$ should be less than about $1 / 100$ of the wavelength.

At $10 \mathrm{GHz}$, the wavelengthis $30 \mathrm{~mm}$ and so the allowable error is $0.3 \mathrm{~mm}$. For a spherical surface of radius $R$, in Ref. 7 , the following relationship is obtained between $\delta_{\text {rms }}$ and the side length $L$ of the triangles:

$$
\delta_{\mathrm{rms}}=L^{2} / 8 \sqrt{15} R
$$

For a shallow paraboloid, the radius of curvature is approximately twice the focal length $F$; thus,

$$
R \approx 2 F
$$

For $D=3.0 \mathrm{~m}$ and $F=0.4 \times D=1.2 \mathrm{~m}$, Eq. (10) can be solved for $L$ and yields

$$
L=0.15 \mathrm{~m}
$$

Thus, the number of triangles across a $3 \mathrm{~m}$ diagonal of the hexagon will be 20, which means that the net will consist of 10 concentric rings of equilateral triangles. (Note that the net of Fig. 5 is formed by three concentric rings.) The corresponding total length of the cables to make up both nets is $\approx 300 \mathrm{~m}$. When it is assumed that the members of the cable nets are made from graphite composite tape $\left(\rho=1740 \mathrm{~kg} / \mathrm{m}^{3}\right)$ with a rectangularcross section of 5.0 by $0.2 \mathrm{~mm}$ and that the weight of the joints in the net and of the tension ties can be accounted for by doubling the density of the tapes, the total mass of the two nets and tension ties is $1.04 \mathrm{~kg}$.

\section{Mesh}

The reflective mesh is knitted gold-plated molybdenum wire with a surface density of $0.025 \mathrm{~kg} / \mathrm{m}^{2}$. This value is doubled to account

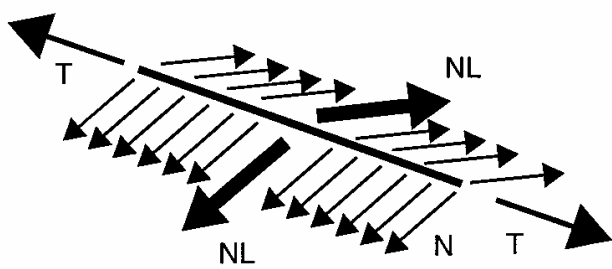

Fig. 19a Loads on cable.

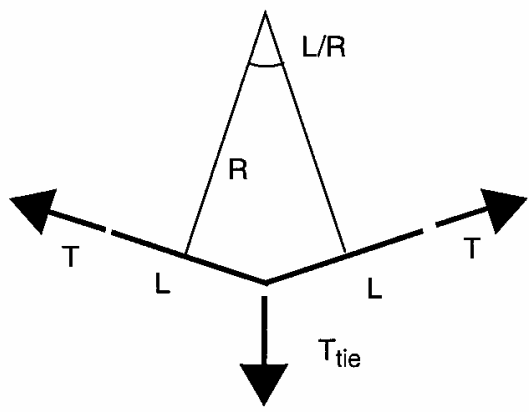

Fig. 19b Equilibrium of a node.

for seams and surface treatment. Approximating the mesh area with the area of a spherical cap, we have

$$
A=2 \pi R H
$$

where $R$ is the radius of the sphere; hence, $R=2 F=2.4 \mathrm{~m}$. $H$ is the height of the cap; hence, $H=0.469 \mathrm{~m}$. Thus, $A=7.07 \mathrm{~m}^{2}$, and the corresponding mass is $0.35 \mathrm{~kg}$.

\section{Force in Springs}

The tension in the mesh applies a lateral loading on the cable net to which it is attached because the mesh forms a small kink, of angle $L / R$, at the crossover between adjacent triangles (Fig. 19a). To prevent the sides of the triangles from becoming significantly distorted, the tension $T$ in the cables of the net must be significantly larger than the transverse load.

For a preliminary estimate, $T$ will be set equal to 10 times the mesh tension $N$ multiplied by the triangle side length $L$. With $N=2.0 \mathrm{~N} / \mathrm{m}$, the required tension in the net cables is $T=3.0 \mathrm{~N}$. The force in the tension ties that is required to obtain the specified tension $T$ in the cables is

$$
T_{\text {tie }}=3 T L / R=3 \times 3.0 \times 0.150 / 2.4=0.56 \mathrm{~N}
$$

(See Fig. 19b, but note that only two of the six cables connected to this node are shown.) Note that the average pressure on the net, given by $T_{\text {tie }}$ over the corresponding area of mesh is $29 \mathrm{~N} / \mathrm{m}^{2}$ (when it is assumed that the surface associated with one node is twice the area of a triangle). This pressure is considerably larger than the self-weight of the mesh under gravity.

\section{Ring Structure}

The cable net analyzed in the section "Configuration of Tensegrity Reflector" consisted of only three rings of triangles, whereas a 3 -m reflector requires 10 rings. A preliminary estimate of the loads transmitted to the ring structure by the full-size net can be obtained by assuming that each cable in the 3 -ring reflector represents $3.3 \mathrm{ca}-$ bles in a 10-ring reflector. Thus, the forces in the supporting structure can be calculated by scaling the forces applied by the three-ring net, which gives equivalent forces of $8 \mathrm{~N}$ in the tension ties. Table 2 lists the forces and lengths of the members of the ring structure.

\section{Design of Struts}

The struts are designed to resist Euler buckling, subject to a minimum slenderness $L_{e} / r=200$. Here, $L_{e}$ is the effective length of the strut and $r$ the radius of gyration. For a thin-walled tube of radius $a$

$$
r=\sqrt{I / A}=\sqrt{\pi a^{3} t / 2 \pi a t}=a / \sqrt{2}
$$


Table 2 Forces and lengths

of elements of ring structure

\begin{tabular}{lcc}
\hline \hline Element & Force, $\mathrm{N}$ & Length, $\mathrm{m}$ \\
\hline Horizontal cable & 310 & 1.50 \\
Lateral cable & 376 & 1.96 \\
Strut & -712 & 2.88 \\
\hline \hline
\end{tabular}

When it is assumed that the struts are pin jointed at both ends, $L_{e}$ is the actual length, given in Table 2 . Hence, $L_{e}=2.88 \mathrm{~m}$, which yields $a>0.0204 \mathrm{~m}$.

Graphite fiber tubes $\left(E=227.5 \mathrm{GN} / \mathrm{m}^{2}\right.$ and $\left.\rho=1740 \mathrm{~kg} / \mathrm{m}^{3}\right)$ with an outer diameter of $42 \mathrm{~mm}$ and wall thickness of $0.5 \mathrm{~mm}$ were selected. Their buckling load is $3.8 \mathrm{kN}$, well above the actual load. The total length of these struts is $17.3 \mathrm{~m}$ and the total mass $1.96 \mathrm{~kg}$.

Each strut must be collapsed to less than a quarter of its length to fit into the launch envelope. The simplest solution at this preliminary stage is to assume that telescopic tubes will be used, for example, the scheme developed in Ref. 8 could be adopted. Hence, the mass estimated earlier is amplified by $50 \%$, to allow for tube overlap and the variation in the cross section of the tubes; the mass of the deploymentmotor, latches, cables, and pulleys is estimated at $0.2 \mathrm{~kg}$ per strut.

\section{Cable Dimensions}

It is assumed that graphite composite tape, with a design strength of $500 \mathrm{~N} / \mathrm{mm}^{2}$, is used also for the cables of the ring structure. The maximum cable force is $1880 \mathrm{~N}$, assuming a factor of safety of 5 . Thus, the required cross-sectional area is $3.76 \mathrm{~mm}^{2}$, and, because the total length of the cables in the ring structure is $29.8 \mathrm{~m}$, their mass is $0.19 \mathrm{~kg}$.

\section{Conclusions}

The proposedreflector conceptmeets the requirementsof a particular small satellite mission, requiring a 3-m aperture with $F / D=0.4$ and operating at $10 \mathrm{GHz}$, which can be packaged as a compact bundle that is less than $0.8 \mathrm{~m}$ high. It was estimated that a surface accuracy of $0.3 \mathrm{~mm}$ is required.

The proposed solution consists of a cable net forming triangles with side length of $0.15 \mathrm{~mm}$ to which the reflective wire mesh is attached. This cable net is prestressed against an identical cable net, as in the AstroMesh reflector. However, there are two key differences between the new concept and the AstroMesh reflector. First, by the use of a tensegrity structure to deploy the cable nets, the need for mechanical joints can be avoided, thus reducing the cost and potentially increasing the geometric accuracy of the structure. Second, the geometric configuration of the structure has been optimized by rotating the two cable nets about the axis of the reflector, which has the effect of substantially reducing the compression carried by the struts of the tensegrity structure. Thus, it has been possible to design a 3-m-diam precision structure with a mass conservatively estimated at $6.3 \mathrm{~kg}$.

The proposed concept has been shown to be feasible by constructing a simple demonstrator, but note that important issues, such as the attachment of the reflector to the spacecraft, the deployment sequence of the reflector, and the feasibility of nonsymmetric (e.g., offset configurations), were not addressed in this study.

More generally, it is noted that cable-and-strut deployable structures, of which the reflector structure concept proposed in this paper is an example, suffer a mass penalty in comparison with articulated structures. The reason for this penalty is that they have to be prestressed after deployment, which leads to an increase in the crosssectional dimensions of the compression members, but this penalty is offset by the reduced cost and mass of the joints. Reducing the additional compression in the struts to a minimum is essential for the proposed approach to be competitive.

\section{Appendix: Cable Net Generation}

This Appendix describes the procedure used for generating the two triangulated cable nets of paraboloidal shape. The procedure is applicable to nets forming any regular, $m$-sided polygon and is illustrated in Fig. A1. The symmetry unit is subdivided into $n \times n$

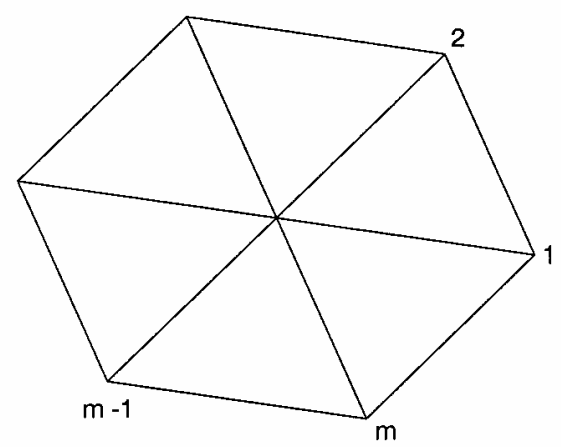

a) $m$-sided polygon

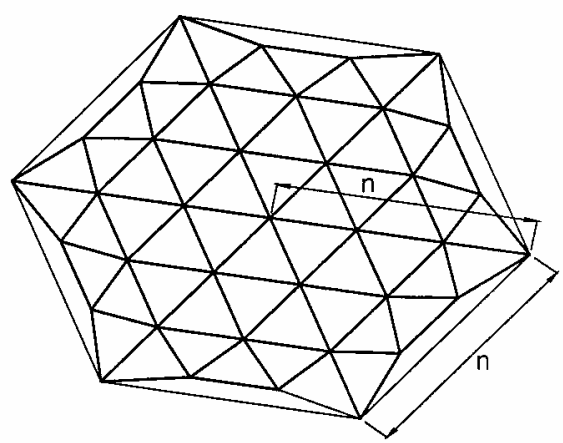

b) Subdivision of order $n$

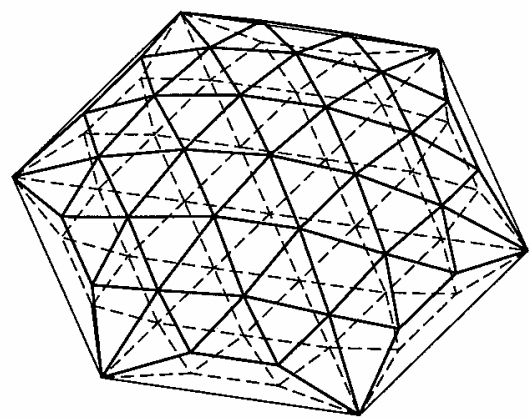

c) Vertical mapping onto paraboloid

Fig. A1 Generation of net.

triangles and all nodes are projected onto the required paraboloidal surface, Fig. A1c.

The total number of triangles $t$, elements $b$, and joints $j$ are, respectively,

$$
\begin{gathered}
t=m n^{2} \\
b=m n(1+3 n) / 2 \\
j=1+m n(1+n) / 2
\end{gathered}
$$

The subdivision is defined by $m, n$, and the circumscribed radius $R$. The edge sag-to-span ratio (Fig. A2) is defined as

$$
\delta / 2 R_{0} \tan \alpha
$$

where $\delta$ is the sag, $\alpha=\pi / m$, and $R_{0}$ is the inscribed radius. Note that the span used in the definition, $2 R_{0} \tan \alpha$, is different from the distance between the outer vertices, which is $2 R \tan \alpha$.

Given the sag-to-span ratio, $R_{0}$ is calculated by subtracting from $R$ the following lengths (Fig. A2):

$$
\begin{gathered}
\Delta_{1}=R \frac{1-\cos \alpha}{\cos \alpha} \\
\Delta_{2}=\frac{\delta}{\cos \alpha}
\end{gathered}
$$




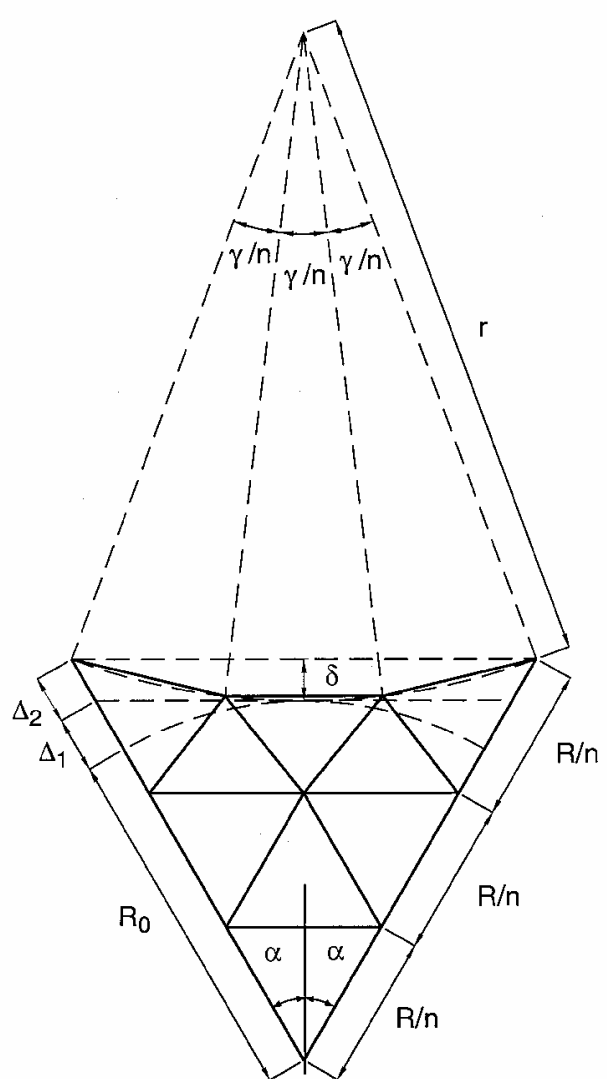

Fig. A2 Triangular subdivision of a sector, with $\boldsymbol{n}=3$.

From Eqs. (A4-A6), the relation between $R$ and $R_{0}$ is written as

$$
\frac{R}{R_{0}}=\frac{1+2 \rho \tan \alpha}{\cos \alpha}
$$

The radius $R$ is divided into $n$ equal parts, corresponding to $n-1$ rings of identical triangles. In the outer ring, the triangles are distorted along the edge. The edge joints are equidistantly positioned on a circular arc with radius $r$ and opening angle $\gamma$ (Fig. A2)

$$
r=\frac{\delta^{2}+R^{2} \sin ^{2} \alpha}{2 \delta}
$$

$$
\gamma=2 \arccos \frac{r-\delta}{r}
$$

The horizontal projection of the length of the edge elements is $2 r \sin (\gamma / 2 n)$. Note that for odd values of $n$ the actual twodimensional sag of the edge elements will be slightly less than $\delta$, as shown in Fig. A2 for $n=3$.

\section{Acknowledgments}

This research was partially supported by the Defence Evaluation Research Agency, under a program sponsored by the British National Space Centre. Financial support from the Royal Swedish Academy of Sciences for A. G. Tibert's visit to the Deployable Structures Laboratory, University of Cambridge, during the year 2000 is gratefully acknowledged.

\section{References}

${ }^{1}$ Thomson, M. W., "The AstroMesh Deployable Reflector," Proceedings of the Fifth InternationalMobile Satellite Conference (IMSC'97), Jet Propulsion Lab., JPL Publ. 97-11, California Inst. of Technology, Pasadena, CA, 1997, pp. 393-398.

${ }^{2}$ Thomson, M. W., "The AstroMesh Deployable Reflector," IUTAM-IASS Symposium on Deployable Structures: Theory and Applications, edited by S. Pellegrino and S. D. Guest, Kluwer Academic, Dordrecht, The Netherlands, 2000, pp. 435-446.

${ }^{3}$ Miura, K., "Concept of Tension Activated Cable Lattice Antenna," International Astronautical Federation, Paper IAF-86-206, Oct. 1986.

${ }^{4}$ Duffy, J., Rooney, J., Knight, B., and Crane, C. D., "A Review of a Family of Self-Deploying Tensegrity Structures with Elastic Ties," Shock and Vibration Digest, Vol. 32, 2000, pp. 100-106.

${ }^{5}$ Calladine, C. R., "Buckminster Fuller's Tensegrity Structures and Clerk Maxwell's Rules for the Construction of Stiff Frames," International Journal of Solids and Structures, Vol. 14, 1978, pp. 161-172.

${ }^{6}$ Pellegrino, S., "Structural Computations with the Singular Value Decomposition of the Equilibrium Matrix," International Journal of Solids and Structures, Vol. 30, 1993, pp. 3025-3035.

${ }^{7}$ Agrawal, P. K., Anderson, M. S., and Card, M. F., "Preliminary Design of Large Reflectors with Flat Facets," IEEE Transactions on Antenna and Propagation, Vol. AP-29, 1981, pp. 688-694.

${ }^{8}$ Becchi, P., and Dell'Amico, S., "Design and Testing of a Deployable, Retrievable Boom for Space Applications," Proceedings of 23rd Aerospace Mechanisms Symposium, NASA CP-3032, Marshall Space Flight Center, Huntsville, AL, 1989, pp. 101-112. 